\title{
NORMAS PERCEBIDAS POR ESTUDANTES UNIVERSITÁRIOS SOBRE O USO DE ÁLCOOL PELOS PARES
}

\author{
Elias Barbosa de Oliveira \\ John Cunningham² \\ Carol Strike \\ Bruna Brands ${ }^{3}$ \\ Maria da Gloria Miotto Wright ${ }^{4}$
}

Oliveira EB, Cunningham J, Strike C, Brands B, Wright MGM. Normas percebidas por estudantes universitários sobre o uso de álcool pelos pares. Rev Latino-am Enfermagem 2009 novembro-dezembro; 17(Esp.):878-85.

O álcool é a droga lícita de maior prevalência entre estudantes universitários e consumida de forma recreacional. Os objetivos deste estudo foram identificar as normas percebidas sobre o uso de álcool entre estudantes universitários, contrastar a percepção dos estudantes em relação ao uso de álcool por seus pares com a atual prevalência do uso de álcool e comparar o próprio uso de álcool com a percepção do uso de álcool pelos pares. Utilizou-se a técnica (survey) com 275 estudantes de uma universidade pública do Rio de Janeiro (Brasil), em 2008. A percepção dos estudantes sobre o uso de álcool pelos pares uma vez na vida e nos últimos doze meses foi acurada, porém, em relação aos últimos trinta dias houve percepção errônea (hiperestimativa). Os estudantes consomem bebidas alcoólicas de forma recreacional, principalmente em festas e bares, em companhia de amigos e pares. O consumo se mostra acima dos padrões estabelecidos.

DESCRITORES: drogas ilícitas; bebidas alcoólicas; estudantes universitários; grupo associado

\section{PERCEIVED NORMS OF PEER ALCOHOL USE AMONG UNIVERSITY STUDENTS}

Alcohol is the most prevalent legal drug among university students, and it is used recreationally. The objectives of this study were to identify the perceived norms of alcohol use among university students, contrast the students' perception regarding peer alcohol use and the actual alcohol use prevalence, and compare the subjects' own alcohol use with their perception of peer alcohol use. A survey was performed on 275 students of a public university located in Rio de Janeiro (Brazil), in 2008. The students' perception about peer alcohol use once in a lifetime and over the last 12 years was correct; but their perception regarding the last 30 days was overestimated. Students consume alcoholic beverages recreationally, mainly at parties and bars with friends and peers. Alcohol use is above established standards.

DESCRIPTORS: street drugs; alcoholic beverages; university students; peer group

\section{NORMAS PERCIBIDAS POR LOS ESTUDIANTES UNIVERSITARIOS SOBRE EL USO DE ALCOHOL ENTRE SUS PARES}

El alcohol es la droga lícita de mayor consumo entre estudiantes universitarios, siendo su consumo considerado como de diversión. Los objetivos de este estudio fueron identificar las normas percibidas sobre el uso del alcohol entre estudiantes universitarios. Se deseaba comparar la percepción que los estudiantes tenían en relación al uso de alcohol entre sus pares con el uso real que los propios estudiantes hacían del alcohol. Se utilizó la técnica de la encuesta en 275 estudiantes, en una universidad pública de Río de Janeiro, en Brasil, en 2008. Se encontró que la percepción de los estudiantes sobre el uso del alcohol entre sus pares, en alguna vez en la vida y en los últimos doce meses, fue exacta, pero en relación a los últimos treinta días tuvieron una percepción errónea, esta fue sobreestimada. También, los estudiantes consumen bebidas alcohólicas para divertirse, principalmente en fiestas y bares en compañía de amigos y pares. Finalmente se encontró que el consumo es mayor que los estándares establecidos. Se recomienda prestar atención a los factores protectores.

DESCRIPTORES: drogas ilícitas; bebidas alcohólicas, estudiantes universitarios; grupo paritário

${ }^{1}$ Enfermeiro, Ph.D., Professor Adjunto, Universidade do Estado do Rio de Janeiro, Brasil, e-mail: eliasbo@tutopia.com.br. ${ }^{2}$ Ph.D., Pesquisador, Health Systems Research and Consulting Unit, Centre for Addiction and Mental Health, CAMH, University of Toronto, Canada, e-mail: john_cunningham@.net, carol_strike@camh.net. ${ }^{3}$ Ph.D., Pesquisador Sênior, Office of Research and Surveillance, Drug Strategy and Controlled Substances Programme, Health Canada and Public Health and Regulatory Policies, Centre for Addiction and Mental Health, CAMH, University of Toronto, Canadá, e-mail: bruna brands@camh.net; ${ }^{4}$ Ph.D., Especialista Sênior, Coordenador, Educational Development Program, Demand Reduction Section, Inter-American Drug Abuse Control Commission, CICAD, Organization of American States, OAS, Estados Unidos, e-mail: gwright@oas.org. 


\section{INTRODUÇÃO}

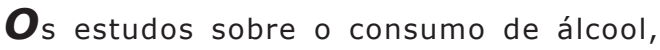
tabaco e drogas ilícitas, entre estudantes universitários, têm aumentado nos últimos anos de forma acelerada na tentativa de compreender as características de consumo e o perfil da população de interesse, visando extrapolar dados para a população geral e aprimorar programas de prevenção existentes em instituições de ensino superior. No entanto, apesar de os estudos transversais serem a metodologia de escolha para a avaliação do consumo de drogas, nesse grupo de indivíduos, infelizmente os trabalhos realizados são poucos e não representativos $^{(1)}$.

O ambiente universitário é um contexto importante para o desenvolvimento dos estudantes nessa fase, pois é fonte de crescimento pessoal e profissional, mas, também, se relaciona a fatores de risco que podem levar os universitários a se engajar em comportamentos que comprometem a saúde, incluindo o uso de $\operatorname{drogas}^{(2)}$.

O consumo de drogas entre estudantes universitários está associado à vida social mais intensa, talvez porque, fora de casa, ou em grupos, o acesso às drogas seja mais fácil ou, então, porque esses indivíduos são, devido à educação que receberam ou ao meio onde estão inseridos, mais "abertos" e com menos "tabus" em relação a esse consumo ${ }^{(3)}$. Dentre os fatores de risco, a influência dos pares é preditor importante para o uso de drogas, como tem sido identificado em vários estudos, sendo que a influência pode ser vista tanto como fator de risco ou de proteção dada a semelhança comportamental entre eles ${ }^{(4)}$.

Uma das mais importantes abordagens que explica a influência dos pares é a teoria das Normas Sociais, que permite predizer os comportamentos dos indivíduos. Dentre os tipos estudados, as normas descritivas, na qual a autoria se apoiou neste estudo, referem-se à percepção de como as pessoas se comportam em determinadas situações. A percepção das normas sociais influencia os comportamentos por meio do processo de comparação social, que serve como padrão pelo qual se avalia e se ajusta o próprio comportamento a fim de alcançar semelhança com o grupo de referência ${ }^{(5)}$.

As normas sociais são construídas por meio de processos inferenciais que partem de dados brutos, a partir de três fontes primárias: comportamentos observáveis, comunicações diretas e indiretas (palavras e ditados) e conhecimento de características pessoais (atitudes e comportamentos). A combinação dessas três fontes para criar as inferências sobre os comportamentos dos outros pode levar a percepções imprecisas das normas ${ }^{(6)}$.

\section{OBJETIVOS}

Identificar as normas percebidas sobre o uso de álcool pelos estudantes universitários, contrastar a percepção dos estudantes em relação ao uso de álcool pelos pares, com a atual estimativa do uso de álcool, e comparar o próprio uso de álcool com a percepção do uso de álcool pelos pares.

\section{METODOLOGIA}

Trata-se de recorte de estudo exploratório, quantitativo, transversal e multicêntrico, que envolveu nove universidades de cinco países da América Latina (Brasil, Colômbia, Chile, Honduras e Peru). A população foi constituída por 275 estudantes universitários na faixa etária de 18 a 24 anos, cursando o segundo e o terceiro ano das carreiras enfermagem, medicina e odontologia, de uma universidade pública, situada no município do Rio de Janeiro.

A coleta de dados ocorreu em três unidades de ensino, no período de março a junho de 2008. Como instrumento, utilizou-se questionário (survey) dividido em quatro partes: o perfil sociodemográfico do grupo, cinco questões sobre a percepção (estimativa) dos estudantes sobre o uso de drogas pelos pares, catorze questões sobre a própria experiência com drogas, questões sobre as consequências do uso de drogas, políticas da universidade a respeito das drogas e acesso às drogas. $O$ instrumento foi adaptado a partir de dois instrumentos frequentemente utilizados para a avaliação das normas percebidas sobre o uso de drogas entre os pares e uso de drogas no contexto universitário do Canadá.

Este estudo seguiu as recomendações éticas fundamentais para garantir segurança aos participantes. Todos os procedimentos foram 
revisados e aprovados pelo Comitê de Revisão Institucional do Center of Addiction and Mental Health (CAMH - University of Toronto) - Protocolo n.339/ 2007 e pelo Conselho Nacional de Ética em Pesquisa (CONEP), em Brasília (DF), por se tratar de projeto multicêntrico, sendo protocolado sob n.951/2008.

No que se referiu à confiabilidade dos dados, além da mesma ser garantida pela Resolução 196/ 96, mediante a assinatura do termo de consentimento livre e esclarecido, enfatizou-se que a identidade dos entrevistados seria preservada e que, em qualquer momento, poderiam desistir da participação no estudo. Esclareceu-se que os resultados seriam divulgados futuramente, através de artigos e apresentação do estudo em eventos, no intuito de contribuir para a pesquisa relacionada ao uso de drogas entre estudantes universitários.

Ao término da coleta, os dados foram armazenados no programa EpiData v 3.1 e $10 \%$ dos dados foram submetidos a controle de qualidade, randomizados e comparados com os questionários. Esse processo foi estendido até não serem encontrados mais erros, sendo os dados exportados para o Statistical Package for the Social Sciences (SPSS).

A fim de contrastar as normas percebidas pelos estudantes universitários sobre o uso de drogas com a estimativa de uso de drogas, foi identificada a diferença entre as normas percebidas sobre uso de drogas pelos pares e a estimativa de uso de drogas entre os estudantes. Norma acurada/norma superestimada/norma subestimada=norma percebida - prevalência do uso de drogas. A prevalência do uso de drogas foi obtida de estudos epidemiológicos mais recentes no país.

Neste artigo estão apresentados somente os resultados sobre o consumo do álcool. Os resultados sobre o uso de outras drogas serão apresentados em outro artigo.

\section{RESULTADOS}

Participaram do estudo 275 estudantes da carreira de enfermagem (Tabela 1), que cursavam o segundo e terceiro ano de graduação $(65,82 \%)$, são do sexo feminino $(82,93 \%)$, solteiros $(95,27 \%)$, encontravam-se na faixa etária de 21 a 22 anos $(46,9 \%)$ e não trabalhavam $(89,09 \%)$.
Tabela 1 - Características sociodemográficas dos estudantes universitários de uma universidade pública. Rio de Janeiro, 2008

\begin{tabular}{lcc}
\hline \multicolumn{1}{c}{ Variáveis } & N & $\%$ \\
\hline Carreiras & 121 & 44 \\
Enfermagem & 78 & 28,4 \\
Medicina & 76 & 27,6 \\
$\quad$ Odontologia & & \\
Ano de escolaridade & 181 & 65,82 \\
$\quad$ Terceiro ano & 94 & 34,18 \\
$\quad$ Segundo ano & & \\
Gênero & 228 & 82,93 \\
Feminino & 47 & 17,97 \\
Masculino & & \\
Idade & 44 & 16 \\
19-20 & 129 & 46,9 \\
$21-22$ & 62 & 22,5 \\
23-24 & 40 & 14,5 \\
$\quad$ 24 & & \\
Condição conjugal & 262 & 95,27 \\
Solteiro & 13 & 4,73 \\
Casado & & 10,91 \\
Trabalhando & 31 & 89,09 \\
Sim & 244 & \\
Não & & \\
\hline
\end{tabular}

$N=275$

Contraste das estimativas do uso de bebidas alcoólicas em relação aos pares e às experiências

No intuito de identificar as normas percebidas pelos estudantes sobre o uso de álcool pelos pares e contrastá-las com as próprias experiências, foram consideradas as seguintes variáveis: estimativa do uso de álcool pelos pares uma vez na vida e nos últimos doze meses, locais onde os pares comumente usam álcool e em companhia de quem as usam. Sobre as próprias experiências, foram avaliadas o número de doses em média que os estudantes consumiram nos últimos doze meses, se beberam cinco ou mais doses em uma única ocasião nos últimos doze meses e o maior número de doses que beberam em uma única ocasião nos últimos trinta dias (uso habitual).

Sobre a percepção do uso de álcool pelos pares, 250 estudantes $(91,25 \%)$ estimaram que os pares consumiram bebidas alcoólicas pelo menos uma vez na vida e 231 estudantes $(84,25 \%)$ nos últimos doze meses. Ao se contrastar a percepção com o uso de álcool pelos próprios estudantes, identificou-se que a estimativa foi acurada, considerando que 240 estudantes $(87,2 \%)$ afirmaram ter ingerido bebidas alcoólicas pelo menos uma vez na vida e 236 estudantes $(85,8 \%)$ as utilizaram nos últimos doze meses. 
Em relação à estimativa e à frequência do consumo de bebidas alcoólicas pelos estudantes nos últimos doze meses (Figura 1), houve estimativa errônea (hiperestimativa) dos estudantes ao afirmaram que os pares consumiram álcool de 2 a 3 vezes por semana, $1 \mathrm{vez}$ na semana e todo dia, tendo ocorrido hipoestimativa nos períodos de 2 a 3 vezes num mês, 1 vez num mês e 1 vez no ano.

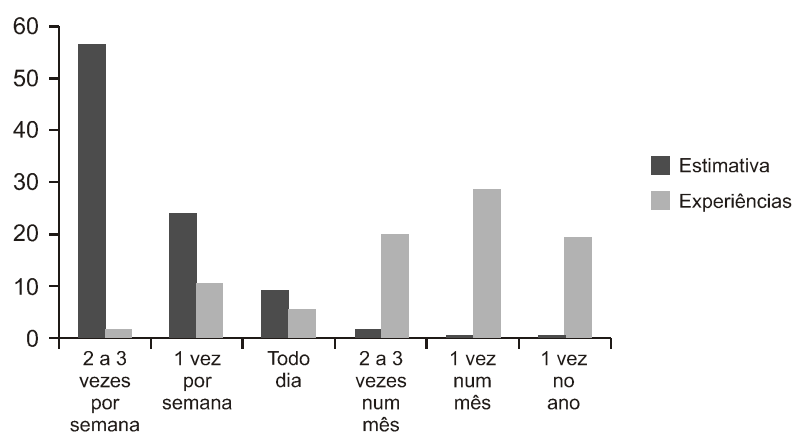

Figura 1 - Contraste da estimativa (percepção) dos estudantes sobre frequência do consumo de álcool pelo pares e as próprias experiências nos últimos doze meses no campo de estudo
Como evidenciado na Figura 2, a estimativa dos estudantes sobre os locais onde os pares consomem bebidas alcoólicas foi acurada em relação a festas e em casa, sendo hiperestimado o consumo em bares, em casa de amigos e no campus.

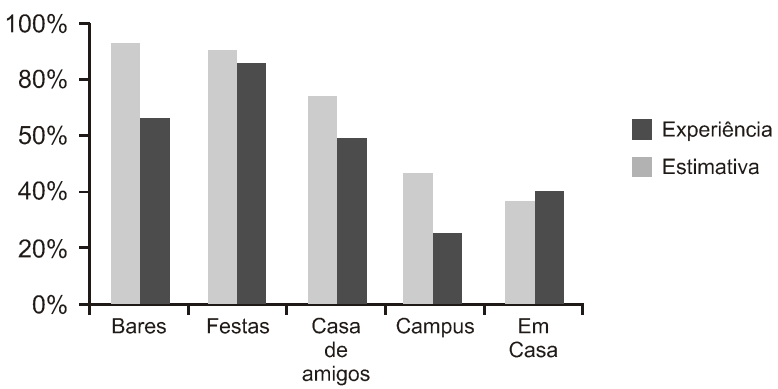

Figura 2 - Contraste da estimativa (percepção) dos estudantes sobre os locais onde os pares consomem álcool e as próprias experiências no campo de estudo

Para os estudantes, os pares consomem bebidas alcoólicas, preferencialmente, em companhia de colegas universitários, amigos, namorado(a), conhecidos, familiares e sozinhos; dados que vão ao encontro das experiências relatadas pelos pares.

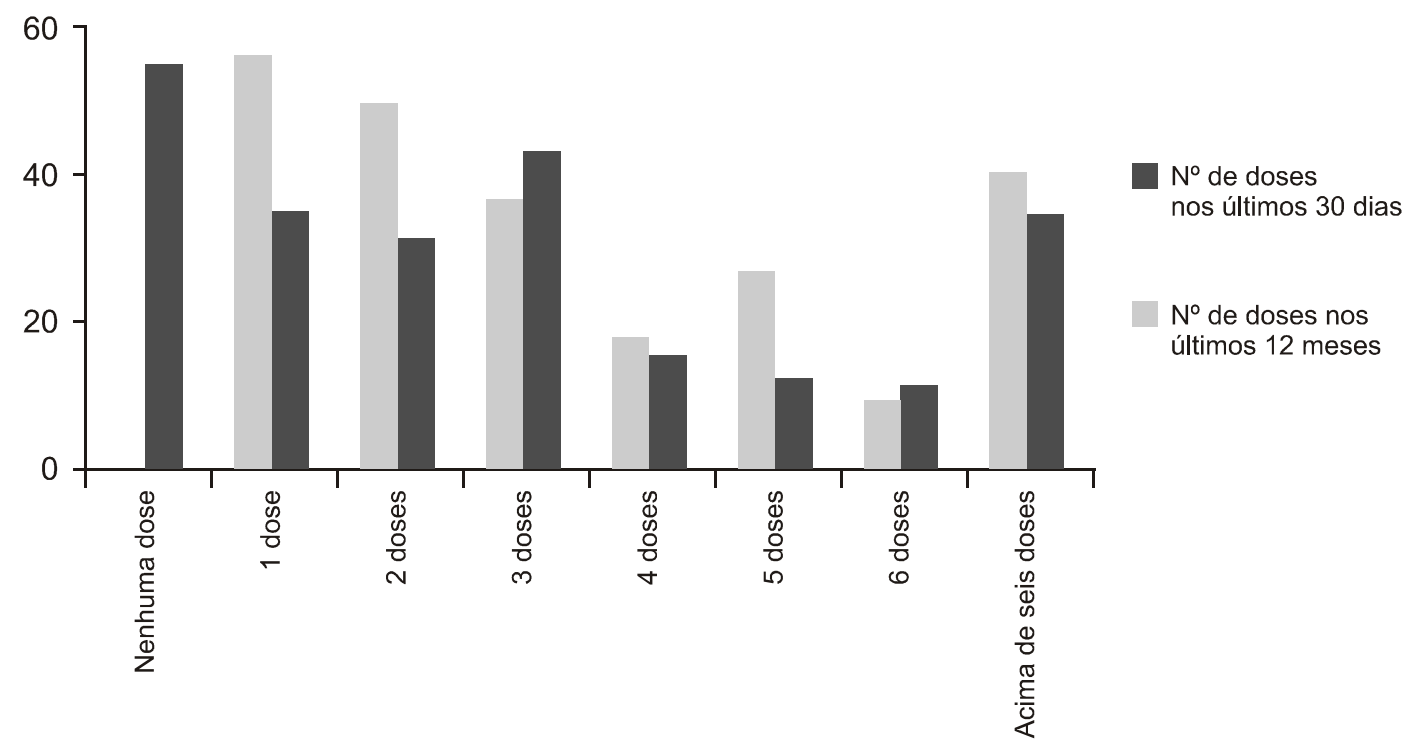

Figura 3 - Número de doses de bebidas alcoólicas consumidas pelos estudantes nos últimos doze meses e nos últimos trinta dias (em uma única ocasião) no campo de estudo

Dos 235 estudantes que consumiram bebidas alcoólicas nos últimos doze meses, 55 negaram o consumo em uma única ocasião (Figura 3) nos últimos trinta dias. No entanto, o consumo de bebidas alcoólicas no mesmo período e nos últimos dozes meses, tendo como referência o índice de consumo
(IC), encontra-se numa faixa considerada de risco, pois $33,8 \%$ dos estudantes consumiram quatro ou mais doses em uma única ocasião, nos últimos doze meses, e 26,9\% nos últimos trinta dias.

O consumo de bebidas alcoólicas pelos pares, segundo 183 estudantes (66,9\%), não interfere nas 
atividades acadêmicas. No entanto, os demais apontaram problemas como: o uso de bebidas alcoólicas bagunça os espaços, atrapalha os estudos, impede a participação em eventos sociais, afeta as atividades esportivas e outras atividades em grupo.

Os principais problemas acarretados aos estudantes, devido ao uso de bebidas alcoólicas, foram: ressaca, náuseas, vômito, os estudantes fizeram algo de que se arrependeram, foram criticados por alguém por terem usado bebidas alcoólicas, tiveram perda da memória, perderam aula, arrumaram briga e/ou discussão, dirigiram sob a influência do álcool, foram feridos, danificaram propriedade, foram mal nas provas e entraram em conflito com a polícia ou outras autoridades.

Apesar dos problemas apontados pelo uso de bebidas alcoólicas, os estudantes sentiram-se relaxados, o álcool ajudou a quebrar o gelo, fez com que se aproximassem das pessoas, ajudou a lidar com o estresse, tornou-os autoconfiantes, o álcool ajudou na tomada de decisões e propiciou raciocínio mais rápido.

\section{DISCUSSÃO}

Características dos sujeitos do estudo

Os estudantes que participaram do estudo fazem parte de uma população considerada de risco para o uso de bebidas alcoólicas, por se tratar de um grupo jovem e viver um momento de transição em suas vidas, principalmente no que se refere às atividades acadêmicas, à dependência familiar, às expectativas em termos de entrada no mercado de trabalho e aos planos futuros. Ressalta-se que, por serem da área da saúde, se encontram em contato mais próximo com a morte, a dor e o sofrimento dos pacientes sob seus cuidados, principalmente na área hospitalar. Tais fatores, em seu conjunto, podem conduzir ao uso de bebidas alcoólicas pelo grupo, como forma de enfrentamento do estresse e alívio da tensão.

Em estudo realizado com acadêmicos de enfermagem, cursando o último período da graduação(7) $^{(7 d e n t i f i c o u-s e ~ q u e ~ o ~ g r u p o ~ r e c o r r e ~ a o ~}$ uso de bebidas alcoólicas e cigarros como válvula de escape ou como forma de relaxar, devido à pressão que sofrem diante das inúmeras atividades de cunho acadêmico, sendo referido o pouco tempo dedicado à família, ao lazer e às demais atividades sociais.
Contrastando as normas percebidas com as experiências em relação ao consumo de bebidas alcoólicas

A estimativa dos estudantes em relação ao consumo de bebidas alcoólicas pelos pares uma vez na vida e nos últimos doze meses, considerando os percentuais $10 \%$ acima (hiperestimativa) e $10 \%$ abaixo (subestimativa), foi acurada, tendo contribuído para essa percepção as disposições internas, as experiências pregressas, as observações e trocas de informações. Há de se considerar também a influência de toda uma conjuntura social, que inclui a família, as políticas antidrogas adotadas pela universidade e a mídia.

Análise das propagandas brasileiras mostra que o marketing das bebidas alcoólicas cria clima normatizador, utilizando estratégias, muitas vezes paradoxais como associar o álcool ao esporte, momentos gloriosos, virilidade e com personalidades de sucesso. A propaganda apela também para momentos de descontração, procurando integrar a bebida à vida dos adolescentes e jovens adultos, como algo adequado e aceitável culturalmente ${ }^{(8)}$.

No que se refere à percepção dos estudantes da frequência do uso de bebidas alcoólicas pelo pares, houve hiperestimativa do consumo semanal, que contrastou com a subestimativa do consumo mensal e anual. Tais estimativas podem estar ancoradas em disposições internas como, também, pelo fato de o campus, onde o estudo foi realizado, possuir, no entorno, inúmeros bares e lanchonetes, onde os estudantes se encontram e consomem bebidas alcoólicas ao término das atividades acadêmicas.

A participação do sexo feminino $(82,91 \%)$ neste estudo é outro fator a ser considerado em relação às estimativas, pois alguns estudos encontraram que as mulheres tendem a superestimar o uso de álcool por seus pares em maior proporção que os homens ${ }^{(9)}$. Outros estudos, no entanto, encontraram que as normas percebidas não variam em função do gênero ${ }^{(10)}$. Encontrou-se, ainda, que mulheres e homens superestimam a quantidade e a frequência de uso de álcool entre os pares de mesmo gênero em maior proporção que entre pares de gênero não específico. Essa superestimação é maior em mulheres que em homens ${ }^{(11)}$.

Os estudantes, em sua maioria, consideraram o acesso às bebidas alcoólicas fácil e muito fácil, sendo adquiridas no campus (53,09\%) e 
fora $(58,9 \%)$ e consumidas em festas, em bares, em casa de amigos, em casa e no campus (provavelmente em encontros e celebrações, pois a venda é proibida na universidade, onde o estudo foi realizado).

$\mathrm{Na}$ percepção dos estudantes, os pares consomem bebidas alcoólicas, principalmente em companhia de colegas universitários e amigos, cuja estimativa foi ratificada a partir das experiências relatadas. O caráter social, recreacional e gregário das bebidas alcoólicas possibilita que os estudantes, a partir de disposições internas, se influenciem mutuamente, estabelecendo normas e padrões característicos de consumo no grupo, ficou evidenciado que apenas $7,3 \%$ dos estudantes consomem bebidas alcoólicas solitariamente.

A população universitária apresenta padrões típicos de uso de álcool e fatores de risco que diferem da população geral, sendo o consumo dessa substância sempre favorecido de forma indireta. Isso porque os universitários se influenciam mutuamente em termos de beber, pela modelagem, pela imitação ou pelo reforço do comportamento de beber. A seleção dos colegas, a escolha do tipo de substância, o padrão de uso e a forma como o consumo de seus pares são percebidos influenciam diretamente no perfil do universitário bebedor e representam fatores de risco importantes ${ }^{(12)}$.

O uso abusivo de bebidas alcoólicas e a exposição aos riscos

O consumo de bebidas alcoólicas pelos estudantes nos últimos doze meses e nos últimos trinta dias foi caracterizado como de uso recreacional, porém, esse padrão de consumo não elimina a possibilidade do uso abusivo, principalmente ao ser identificado que houve consumo excessivo de bebidas alcoólicas (beber problemático). O IC (indicador do consumo) excessivo de bebidas alcoólicas mede a frequência de indivíduos que, nos últimos trinta dias, ingeriram mais de 4 doses. Considera-se dose de bebida alcoólica uma dose de bebida destilada, uma lata de cerveja ou uma taça de vinho ${ }^{(13)}$.

Os estudantes estão ingerindo bebidas alcoólicas acima dos padrões usuais (beber problemático $)^{(14-16)}$ e, em sua maioria, desconhecem a existência de políticas preventivas por parte da universidade em relação às bebidas alcoólicas. Tais dados são preocupantes, pois existem estudos que colocam a problemática do hábito de beber pesado entre estudantes (binge drink), principalmente nos finais de semana, expondo o grupo a riscos como acidentes, violência, morte prematura, doenças sexualmente transmissíveis e gravidez indesejada com altos custos para o indivíduo e sociedade.

Atualmente, a Organização Mundial de Saúde defende a adoção de medidas restritivas ao consumo de bebidas alcoólicas como o aumento do preço das bebidas e a elevação de impostos para reduzir o risco de abuso do álcool. Uma das medidas propostas pelo Centro Brasileiro de Informações sobre Drogas (Cebrid) é a adoção de política de redução de danos, com o objetivo de diminuir o acesso à bebida. Entre as sugestões do grupo interministerial está o incentivo à restrição do horário de funcionamento de bares. Em algumas cidades que adotaram essa medida, como Diadema, SP, conhecida como a cidade mais violenta do Brasil, houve redução significativa nos casos de homicídio ${ }^{(17)}$.

Interferência na vida e problemas acarretados pelo uso de álcool

Além dos efeitos que o uso abusivo de álcool acarreta à saúde do indivíduo, em longo prazo, existem aqueles de ordem psicossocial, identificados no estudo como os prejuízos acarretados às atividades acadêmicas, o fato de dirigirem sob o efeito do álcool e o envolvimento dos estudantes em situações de violência. Portanto, medidas restritivas, como a adotada recentemente no país, que limita o número de doses de bebidas alcoólicas para os condutores de veículos, é um avanço, considerando os riscos e problemas como acidentes, conflitos com a polícia e mortes previsíveis. No Brasil, o consumo de álcool é o que mais preocupa a Organização Mundial de Saúde que diz não ter como exigir, mas pode indicar ao governo que o primeiro caminho é controlar a publicidade sobre bebidas alcoólicas, o que vem sendo realizado a partir da criação da Lei no 9.294, de 15 de julho de 1996, que regulamenta os anúncios de bebidas alcoólicas ${ }^{(18)}$.

Quanto à violência, sabe-se que ela está presente em usuários de drogas ilícitas e consumidores de álcool. Entre universitários, o comportamento agressivo não é identificado somente entre os dependentes, ele também pode ser encontrado em consumidores ocasionais de bebidas alcoólicas, pois os estudantes já dirigiram sob o efeito do álcool e brigaram por estarem sob efeito de alguma substância 
psicoativa. Trata-se de um grupo especial, principalmente por representar o futuro desenvolvimento da sociedade ${ }^{(19)}$.

Quanto às próprias experiências, identificouse que os estudantes fazem uso recreacional de álcool. Porém, em uma mesma ocasião, os dados indicaram que os estudantes consumiram mais de três doses (consumo excessivo), com prejuízos para o desempenho acadêmico e exposição aos riscos como dirigir sob a influência do álcool e envolver-se em situações de violência.

\section{CONCLUSÕES}

As normas percebidas pelos estudantes universitários sobre o uso de álcool refletem a influência dos pares, através de processo de familiarização em relação a padrões de consumo estabelecidos no grupo. Tais padrões resultam de escolhas (seleção), de vivências, da troca de informações e observações do comportamento do outro. Além desse processo, deve-se considerar a vulnerabilidade do grupo devido a fatores como a transição do sistema escolar anterior, o desafio de novas demandas acadêmicas e o fácil acesso ao álcool.

Ao se contrastar a percepção dos estudantes em relação ao uso de álcool por seus pares na vida e nos últimos doze meses, identificou-se que a percepção (estimativa) foi acurada, considerando-se a prevalência do uso atual nesse grupo. No entanto, em relação ao uso habitual (nos últimos trinta dias), a percepção foi equivocada, pois os estudantes hiperestimaram o uso de álcool pelos pares. Inferese que essa percepção pode estar ancorada em disposições internas dos estudantes, ao invés de considerar as características do contexto, devido à falta de informação sobre o uso real de seus pares.

Para os estudantes, tanto em termos de estimativa (percepção) quanto em relação às próprias experiências, o álcool é uma droga de fácil acesso, adquirida tanto fora quanto no campus, sendo consumida principalmente em festas, em bares e em companhia de amigos e colegas universitários.

A maioria dos estudantes relatou desconhecer a existência de políticas preventivas, adotadas pela universidade, em relação ao uso de bebidas alcoólicas. Portanto, considerando os problemas identificados, há necessidade de fortalecimento e incremento de políticas preventivas por parte das entidades governamentais, universidades e profissionais, pois o uso de bebidas alcoólicas traz prejuízos para as atividades acadêmicas e expõe o grupo a riscos psicossociais.

Tendo em vista a limitação da amostra, o que impossibilitou a generalização dos resultados e a inexistência de estudos no país, que discutam a influência dos pares em relação ao uso de álcool com o suporte das normas sociais descritivas, recomenda-se a continuidade de estudos nessa vertente com número maior de estudantes, inclusive de outras áreas.

\section{AGRADECIMENTOS}

Esta pesquisa foi realizada com o apoio, assessoria e patrocínio do governo do Canadá, da Organização dos Estados Americanos (OEA), da Comissão Inter-Americana para o Controle e Abuso de Drogas (CICAD), e do Centro de Drogas e Saúde Mental (CAMH), Canadá. Agradecemos a colaboração de outros colegas que contribuíram de forma direta ou indireta na realização deste estudo.

\section{REFERÊNCIAS}

1. Wagner GA, Andrade AG. Uso de álcool, tabaco e outras drogas entre estudantes universitários brasileiros. Rev Psiquiatr Clin 2008; 35(número especial):48-54.

2. Maggs JL. Alcohol use and binge drinking as goal - directed action during the transition to postsecondary education. In: Schulenberg J, Maggs JL, Hurrelmann K, organizadores. Health risk and developmental transitions during adolescence. Inglaterra (UK): Cambridge University Press; 2007. p. 345-71.

3. Barrias ACR, Queiroz S, Niscadri S, Andrade AG. Comportamento do universitário da área biológica da Universidade de São Paulo em relação ao uso de drogas. Rev
Bras Psiquiatr 2000; 27(4):215-24.

4. Andrews J, Tidesley E, Hops H, Li F. The influence of peers on young adult substance use. Health Psychol J 2002; 21(4):349-57.

5. Mathers CD, Lopez A, Stein C, Fat DM, Rao C, Inoue M, et al. Deaths and disease burden by cause: global burden of disease estimates for 2001. Washington, D.C.: World Bank, Disease Control Priorities Project; 2005 January. (Disease Control Priorities Project Working Paper n. 18).

6. Miller $D$, Prentice $D$. The construction of social norms and standards. In: Higgins T, Kruglanski A. Social psychology handbook of basic principles. New York: Guilford Press; 1996. p. $18 ; 253-75$. 
7. Oliveira EB, Furegato ARF. O trabalho do acadêmico de enfermagem como fator de risco para o consumo de álcool e outras drogas. Rev Latino-am Enfermagem 2008; 16(n. esp):565-71.

8. Faller S, Bumaguin DB, Rodrigues VS, Costa C, Pinsky I, Pechansky F. O consumo de álcool influencia a opinião de profissionais de saúde sobre a propaganda de bebidas alcoólicas no Brasil? Rev Psiquiatr Hoje 2007; 29(2):24-7.

9. Borsari B, Carey KB. Descriptive and injunctive norms in college drinking: A meta-analytic integration. J Stud Alcohol 2003; 64(3):331-41.

10. Read JP, Wood MD, Davidoff OJ, McLacken J, Campbell JF. Making the transition from high school to college: The role of alcohol-related social influence factors in students' drinking. Substance Abuse J 2002; 23(1):53-65.

11. Lewis M.A, Neighbors C. Gender specific misperception of college students drinking norms. Psychol Addict Behav 2004; 18(4):334-9.

12. Chavez KAP, O' Brien B, Pillon SC.Uso de drogas e comportamento de risco no contexto de uma comunidade universitária. Rev Latino-am Enfermagem 2005; 13(n. esp):1194-200.

13. Ministério da Saúde (BR). Vigilância de fatores de risco e proteção para doenças crônicas por inquérito telefônico Vigitel [site na internet] março 2006 [Acesso out 2008]. Disponível em: http://bvsms.saude.gov.br/bvs/publicacoes/relatorio _vigitel.pdf

14. Santos SMS, Oliveira MLF. Knowledge about Aids and drugs among undergraduate students in a higher education institution in the State of Paraná. Rev Latino-am Enfermagem 2009; 17(4):522-8.

15. Barroso T, Mendes A, Barbosa A. Analysis of the alcohol consumption phenomenon among adolescents: study carried out with adolescents in intermediate public education. Rev Latino-am Enfermagem 2009; 17(3):347-53.

16. Jordán Jinez ML, Souza JRM, Pillon SC. Drug use and risk factors among secondary students. Rev Latino-am Enfermagem 2009; 17(2):246-52.

17. Peuker AC, Fogaça J, Bizarro L. Expectativas e beber problemático entre estudantes universitários. Psicol: Teoria Pesqui 2006; 22(2):193-200.

18. Lei no 9.294, de 15 de julho de 1996. Dispõe sobre as restrições ao uso e à propaganda de produtos fumígeros, bebidas alcoólicas, medicamentos, terapias e defensivos agrícolas [site na internet] julho 1996 [Acesso agosto 2008]. Disponível em: http://e-legis.anvisa.gov.br/leisref/public/ showAct. php?id=264

19. Ministério da Saúde (BR). Relatório sobre Neurociência do Uso e Dependência de Drogas da Organização Mundial de Saúde [site na internet] 2004. [Acesso agosto 2008]. Disponível em: http://portal.saúde.Senad.gov.br 